\title{
Identification of key proteins and IncRNAs in hypertrophic cardiomyopathy by integrated network analysis
}

Xiaofeng Hu², Guilin Shen², Xiaoli Lu², Guomin Ding², Lishui Shen ${ }^{1}$

${ }^{1}$ Department of Cardiology, Zhejiang Hospital, Hangzhou, Zhejiang Province, China 'Department of Cardiology, Anji People's Hospital, Huzhou, Zhejiang Province, China

Submitted: 18 December 2017

Accepted: 2 April 2018

Arch Med Sci 2019; 15, 2: 484-497

DOI: https://doi.org/10.5114/aoms.2018.75593

Copyright (c) 2018 Termedia \& Banach

\section{Abstract}

Introduction: Hypertrophic cardiomyopathy (HCM), a genetically heterogeneous disorder of cardiac myocytes, is one of the main causes of sudden cardiac death of young people. However, the molecular mechanism involved in HCM has remained largely unclear. Of note, non-coding RNAs were reported to play an important role in human diseases. In this study, we focused on identifying differentially expressed long non-coding RNA (IncRNAs) and mRNAs in HCM by analyzing a public dataset (GSE36961).

Material and methods: We performed bioinformatics analysis to explore key pathways underlying HCM progression. Gene Ontology (GO) analysis was first performed to evaluate the potential roles of differentially expressed genes and IncRNAs in HCM. Moreover, protein-protein interaction (PPI) networks were constructed to reveal interactions among differentially expressed proteins. Specifically, co-expression networks were also constructed to identify hub IncRNAs in HCM.

Results: A total of 6147 mRNAs $(p<0.001)$ and $126 \operatorname{lncRNAs}(p<0.001)$ were found to be dysregulated in HCM. Gene Ontology (GO) analysis showed that these differentially expressed genes and IncRNAs were associated with metabolism, energy pathways, signal transduction, and cell communication. Moreover, TSPYL3, LOC401431, LOC158376, LOC606724, PDIA3P and LOH3CR2A $(p<0.001)$ were identified as key IncRNAs in HCM progression. Conclusions: Taken together, our analysis revealed a series of IncRNAs and mRNAs that were differentially expressed in HCM and which were involved in HCM progression by regulating pathways, such as metabolism, energy pathways, signal transduction, and cell communication. This study will provide useful information to explore the mechanisms underlying HCM progression and to provide potential candidate biomarkers for diagnosis in HCM.

Key words: differentially expressed gene, long non-coding RNA, hypertrophic cardiomyopathy, protein-protein interaction analysis, co-expression analysis.

\section{Introduction}

Hypertrophic cardiomyopathy ( $\mathrm{HCM}$ ) is a genetically heterogeneous disorder of cardiac myocytes with an estimated prevalence of $0.2 \%$ in the general population [1]. Hypertrophic cardiomyopathy has been one of the main reasons for sudden cardiac death of young people [2]. Clinically, myocyte hypertrophy, disarray, and interstitial fibrosis are the common pathological features [3]. Of note, about $25 \%$ of HCM patients demonstrate left ventricular outflow tract obstruction [4]. Recent studies have

\author{
Corresponding author: \\ Dr. Lishui Shen \\ Department of Cardiology \\ Zhejiang Hospital \\ Hangzhou, 310013 \\ Zhejiang Province, China \\ Phone/fax: \\ +8657187987534 \\ E-mail: heart0571@163.com
}


revealed several genes and pathways associated with HCM progression. For example, MYH7 and MYBPC3 mutations were the two most common mutations in HCM and were found in about half of the patients with familial HCM [5]. Baertling et al. found that mutations in COA6 could cause neonatal hypertrophic cardiomyopathy [6]. Moreover, the Hippo/YAP pathway was found to be activated in HCM [7]. However, the mechanism underlying HCM progression remained unclear. Therefore, there was still an urgent need to identify novel regulators to understand the molecular mechanism involved in HCM.

Long non-coding RNAs (InCRNA) are a novel class of noncoding RNAs longer than 200 nucleotides without protein-coding function [8]. LncRNAs were reported to regulate target genes at transcriptional, post-transcriptional, and post-translational regulation levels in different types of human diseases, such as cancer, acute myocardial infarction and osteoarthritis [9]. In HCM, several studies have focused on the potential roles of IncRNAs. For example, Kitow et al. [10] found that mitochondrial IncRNAs functioned as blood-based biomarkers for cardiac remodeling in HCM patients. Yang et al. [11] also identified 965 up-regulated and 461 down-regulated IncRNAs in HCM. However, these reports just focused on differentially expressed IncRNAs in a small sample size.

In this study, we analyzed a public dataset (GSE36961) to identify differentially expressed IncRNAs and mRNAs in HCM. Protein-protein interaction (PPI) networks were constructed to reveal potential roles of differentially expressed proteins. Furthermore, co-expression analysis was performed to identify hub IncRNAs in HCM. This study will provide useful information to explore the potential biomarkers for diagnosis and prognosis in HCM.

\section{Material and methods}

\section{Microarray data}

The microarray data of GSE36961 contributed by Suresh et al. [12] were downloaded from the National Center of Biotechnology Information (NCBI) Gene Expression Omnibus database (GEO, http://www.ncbi.nlm.nih.gov/geo/). A total of 39 control samples and $106 \mathrm{HCM}$ samples were included in this dataset.

\section{LncRNA classification pipeline}

In present study, we identified differentially expressed IncRNAs in HCM by adopting criteria reported by Zhang et al. [13]. Briefly, first, the GPL570 platform of Affymetrix Human Genome U133 Plus 2.0 Array (Affymetrix Inc., Santa Clara,
California, USA) probe set ID was mapped to the NetAffx Annotation Files (HG-U133 Plus 2.0 Annotations, CSV format, release $31,08 / 23 / 10$ ). The annotations included the probe set ID, gene symbol, and Refseq transcript ID. Second, the probe sets that were assigned a Refseq transcript ID in the NetAffx annotations were extracted. In this study, we only retained those labeled as "NR_" (NR indicates non-coding RNA in the Refseq database). Finally, 2448 annotated IncRNA transcripts with corresponding Affymetrix probe IDs were generated.

\section{Differentially expressed genes}

The raw datasets of HCM from GEO were downloaded and preprocessed by log2 transformation. All sample data were normalized using the limma package in R software version 3.3.0 (https:// www.r-project.org/). The differentially expressed mRNA and IncRNAs were identified by the linear models for microarray analysis (Limma) method [14]. The differentially expressed genes (DEGs) were obtained with thresholds of $|\log F C|>1.5$ and $p<0.001$.

The hierarchical cluster analysis of differentially expressed mRNAs and IncRNAs was performed using CLUSTER 3.0, and the hierarchical clustering heat map was visualized by Tree View [15].

\section{Construction of PPI network and module analysis}

In order to predict protein interactions, which include physical and functional associations, the present study used the Search Tool for the Retrieval of Interacting Genes (STRING) to construct the PPI network for DEGs (minimum required interaction score > 0.4) [16]. In addition, Cytoscape software version 3.4.0 (http://cytoscape. org/download_old_versions.html) was used for visualization of the PPI networks [17]. Following the construction of the PPI network, a module analysis of the network was performed using the Mcode plugin (degree cut-off $\geq 2$ and the nodes with edges $\geq 2$-core) [18]. Additionally, the Network Analyzer was used to compute the basic properties of the PPI network, including average clustering coefficient distribution, closeness centrality, average neighborhood connectivity, node degree distribution, shortest path length distribution, and topological coefficients [19].

\section{Co-expression network construction and analysis}

In this study, the Pearson correlation coefficient of DEG-IncRNA pairs was calculated according to their expression value. The co-expressed DEGIncRNA pairs with the absolute value of Pearson's 
correlation coefficient $\geq 0.6$ were selected and the co-expression network was established using Cytoscape software.

\section{GO and KEGG pathway analysis}

To identify functions of DEGs in HCM, we performed GO function enrichment analysis in 3 functional ontologies: biological process (BP), cellular component (CC) and molecular function (MF) [20]. KEGG pathway enrichment analysis was also performed to identify pathways enriched in HCM using the MAS system (Molecule Annotation System, http://bioinfo.capitalbio.com/mas3/). A p-value less than 0.05 was considered as significant.

\section{Statistical analysis}

The numerical data were presented as mean \pm standard deviation (SD) of at least three determinations. Statistical comparisons between groups of normalized data were performed using the $t$-test or Mann-Whitney $U$-test according to the test condition. A $p<0.05$ was considered statistically significant with a $95 \%$ confidence level.

\section{Results}

\section{Identification of DEGs and IncRNAs in HCM}

In the present study, we analyzed a public HCM expression profiling dataset (GSE36961) to identify DEGs. A total of 39 control samples and 106 HCM samples were included in GSE36961 (Supplementary Table I). We identified 6158 mRNAs with differential expression $(p<0.001)$ between HCM samples and normal tissues. Of these DEGs, 3127 mRNAs were up-regulated and 3021 mRNAs were down-regulated in HCM compared to control samples. Hierarchical clustering analysis of the DEGs in HCM is shown in Figure $1 \mathrm{~A}$.

\section{Functional annotation of DEGs in HCM}

Furthermore, we performed GO analysis for differentially expressed mRNAs. GO analysis showed that the up-regulated DEGs were mainly involved in regulating Regulation of nucleobase metabolism, Metabolism, Energy pathways, Muscle contraction, Embryonic development, Cell growth and/or maintenance, Protein metabolism, and Synapse organization and biogenesis (Figure 1 B).

Meanwhile, the down-regulated DEGs were associated with Signal transduction, Cell communication, Immune response, Metabolism, Energy pathways, Transport, Apoptosis, Protein metabolism, Anti-apoptosis, Regulation of cell cycle, Inflammatory response, Lipid transport, Regulation of immune response, Regulation of gene expression, epigenetic, and Regulation of cell proliferation (Figure $1 \mathrm{C}$ ).

\section{PPI network analysis of DEGs in HCM}

The STRING database was used to predict the interaction between 3127 up- and 3021 down-regulated proteins in HCM (combined score >0.7). We found that the up-regulated PPI network in cluded 1232 nodes and 5261 edges (Figure 2), and the down-regulated PPI network included 1447 nodes and 8751 edges (Figure 3). Several proteins were found to play more important roles in HCM and interacted with more than 50 proteins. The nodes that had higher degrees included up-regulated UBB (degree $=120)$, POLR2C (degree $=71$ ), POLR2L (degree $=69)$, POLR2B (degree $=68)$, and POLR2G (degree $=63$ ) and down-regulated IL-6 (degree $=90)$, JUN (degree $=79)$, ACTB (degree $=$ 78), MAPK3 (degree $=76)$, and MYC (degree $=69$ ). Finally, the PPI network was constructed using Cytoscape and is shown in Figure 3.

Following the construction of the PPI network, a module analysis of the network was performed using the Mcode plugin (degree cut-off $\geq 3$ and the nodes with edges $\geq 3$-core). A total of 35 hubnetworks were identified in the up-regulated DEG mediated PPI networks and 34 hub-networks were identified in the down-regulated DEG-mediated PPI networks.

The top 4 hub-networks in up-regulated DEGmediated PPI networks are shown in Figure 4 Hub-network 1 held 44 nodes and 946 edges (Figure 4 A), hub-network 2 contained 47 nodes and 552 edges (Figure 4 B), hub-network 3 had 15 nodes and 105 edges (Figure $4 \mathrm{C}$ ), and hub-network 4 had 13 nodes and 164 edges (Figure 4 D).

The top 4 hub-networks in down-regulated DEGmediated PPI networks are shown in Figure 5. Hub-network 1 held 122 nodes and 1664 edges (Figure $5 \mathrm{~A}$ ), hub-network 2 contained 22 nodes and 231 edges (Figure 5 B), hub-network 3 had 14 nodes and 90 edges (Figure $5 \mathrm{C}$ ), and hub-network 4 had 38 nodes and 245 edges (Figure 5 D).

\section{Co-expression network analysis of differentially expressed IncRNA in HCM}

Of note, we observed a series of IncRNAs that were differentially expressed in HCM by applying the LncRNA classification pipeline. However, the function of most dysregulated IncRNAs in HCM remained unclear. A total of 1423 IncRNAs were identified in GSE36961. Of these IncRNAs, 67 IncRNAs were up-regulated and 59 IncRNAs were down-regulated in HCM compared to control samples with a $p<0.001$ (Figure $6 \mathrm{~A}$ ).

Then, we constructed a co-expression network of IncRNAs to reveal their potential roles in HCM. By calculating the Pearson correlation coefficient of InCRNA-DEG pairs, we then selected the co-expressed DEG-IncRNA pairs with the absolute value of Pear- 
A

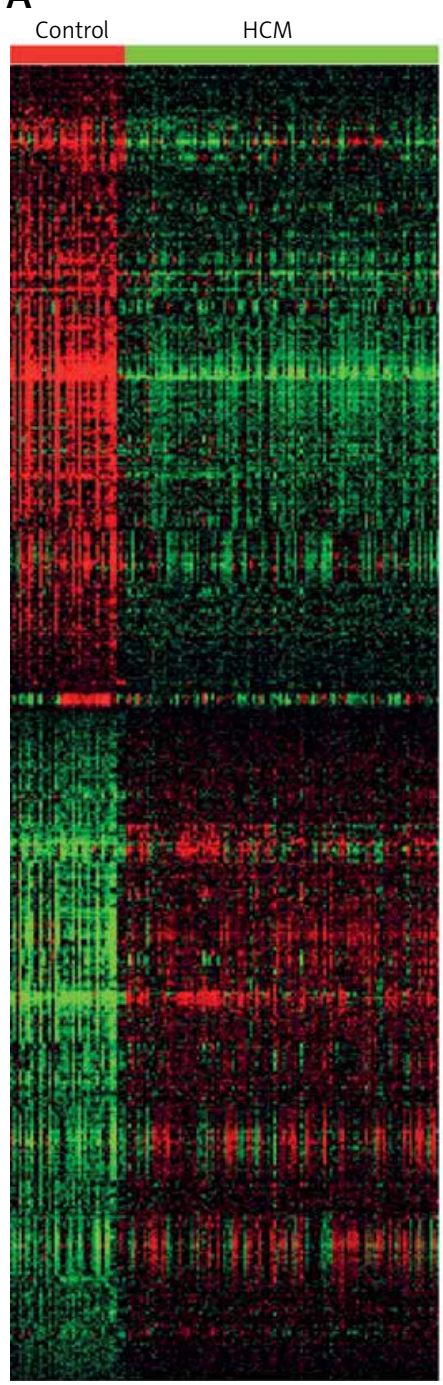

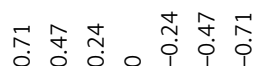

B

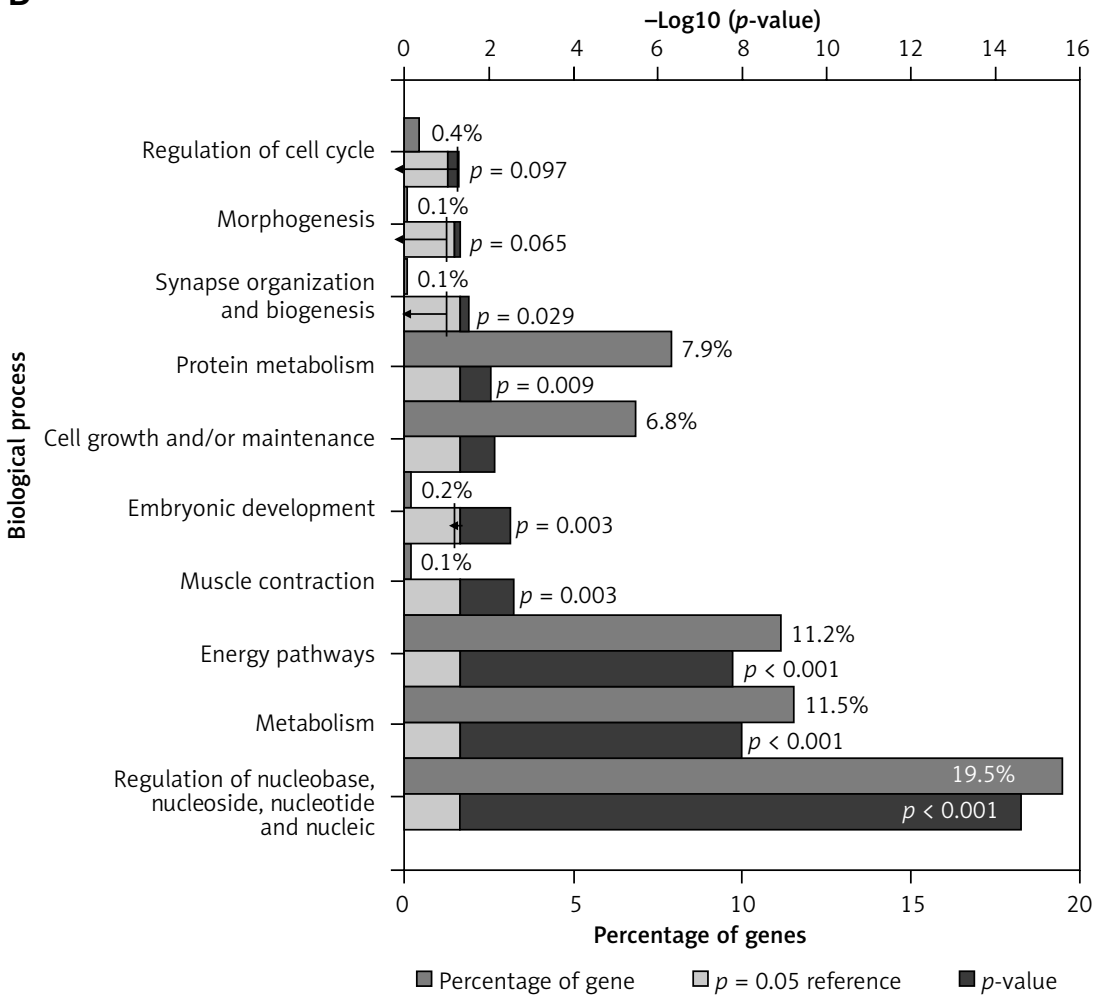

C

Biological processes of down-regulated proteins in HCM

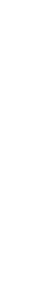

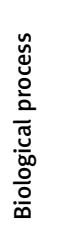

Figure 1. Identification of DEGs and IncRNAs in HCM. A - Hierarchical clustering analysis shows differential gene expression in the HCM by using GSE36961. DEG analysis shows 3127 up-regulated mRNAs and 3021 down-regulated mRNAs in HCM. B - GO analysis shows up-regulated DEG associated biological processes in HCM. C - GO analysis shows down-regulated DEG associated biological processes in HCM 


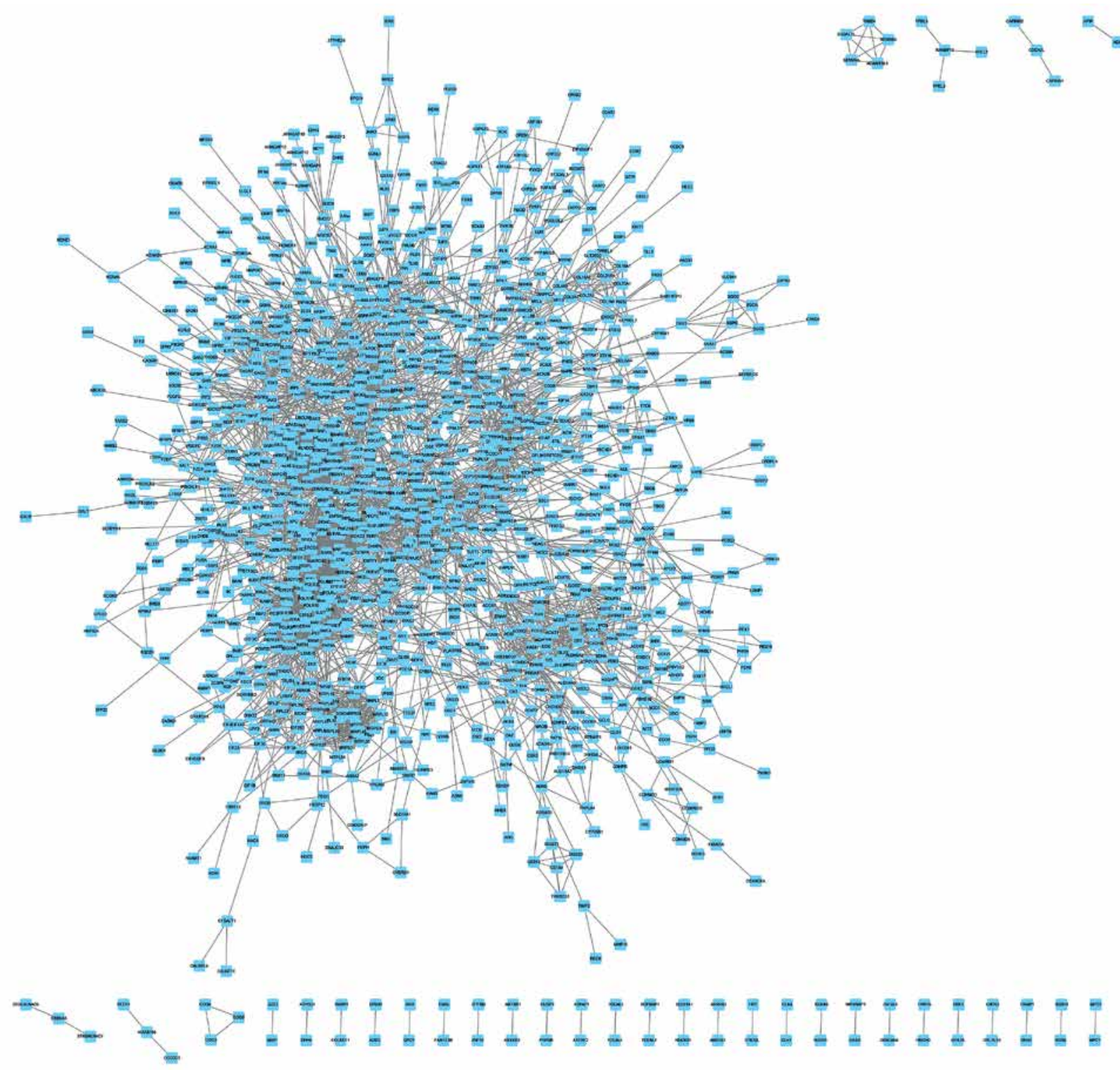

Figure 2. Up-regulated PPI network analysis of DEGs in HCM. We constructed a PPI network for up-regulated proteins in HCM. We found that the up-regulated PPI network included 1232 nodes and 5261 edges

son's correlation coefficient $\geq 0.60$ for co-expression network construction. As shown in Figure 7, a total of 64 IncRNAs and 1892 DEGs were included in the dysregulated IncRNAs associated network.

Furthermore, we performed bioinformatics analysis for differentially expressed IncRNAs. GO analysis showed that the dysregulated IncRNAs were mainly involved in regulating Metabolism, Energy pathways, Regulation of cell proliferation, Transport, Signal transduction, Protein metabolism, Cell growth and/ or maintenance, Regulation of cell cycle, Cell communication, Apoptosis, Regulation of immune response, and Immune response (Figure 6 B).

\section{Identification of key IncRNAs in HCM}

In the co-expression network, we identified two up-regulated IncRNAs (TSPYL3 and LOC401431) and three down-regulated IncRNAs (LOC158376, LOC606724, PDIA3P and LOH3CR2A) as key IncRNAs in HCM. We found these IncRNAs co-expressed with more than 200 mRNAs. Of note, TSPYL3 showed the most important regulating roles by co-expressing with 576 proteins.

To further explore the key IncRNAs in regulating key biological processes in HCM, we constructed IncRNA mediated mRNA-KEGG pathway networks by applying ClueGO in Cytoscape software. We observed that TSPYL3 was associated with the HIF-1 signaling pathway, Complement and coagulation cascades, Gap junction, Apoptosis, Phagosome, and Mineral absorption (Figure 8 A). LOC401431 (Figure 8 B) and LOC158376 (Figure 9 D) were found to be involved in Phagosome regulation. PDIA3P was associated with Complement and co- 


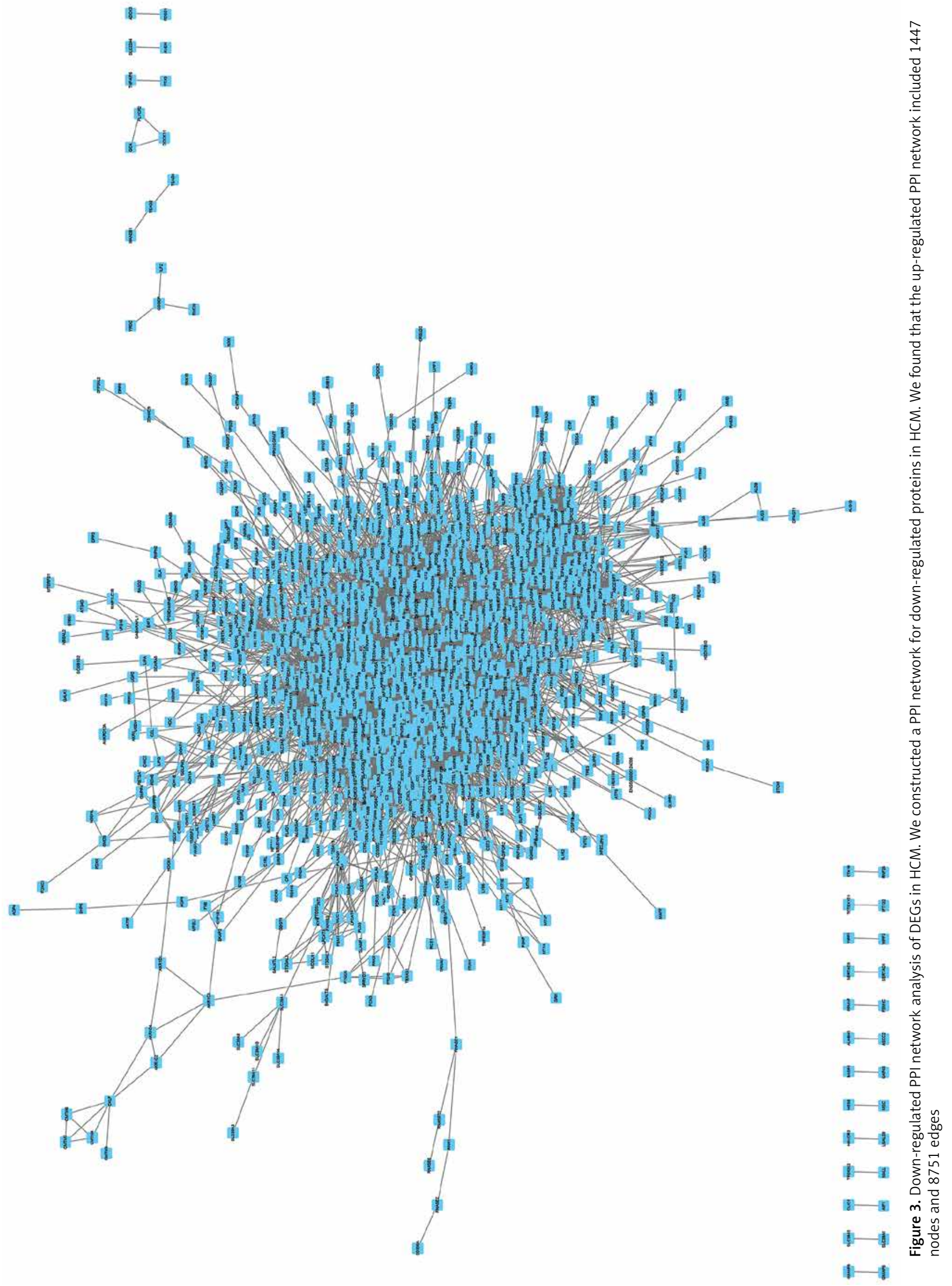



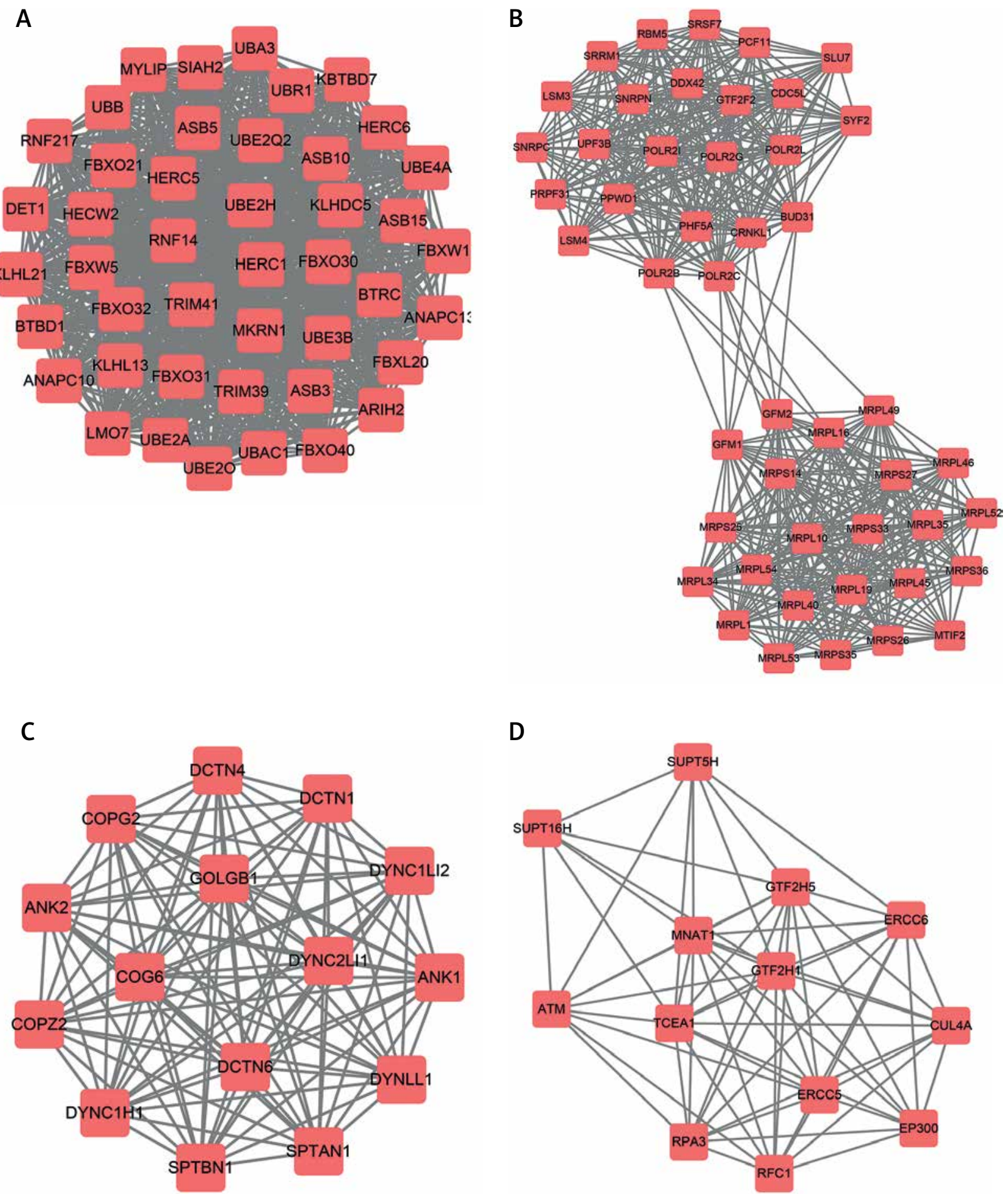

Figure 4. Hub-networks analysis of up-regulated DEGs in HCM. A - Hub-network 1 held 44 nodes and 946 edges. B - Hub-network 2 contained 47 nodes and 552 edges. C - Hub-network 3 had 15 nodes and 105 edges. D - Hub-network 4 had 13 nodes and 164 edges

agulation cascades, Chemokine pathway, Phagosome and Protein processing in endoplasmic reticulum (Figure 9 A). LOH3CR2A was associated with Protein processing in endoplasmic reticulum, Phagosome, Shigellosis, Pathogenic Escherichia coli infection and Proteoglycans in cancer (Figure 9 B). LOC606724 was found to be involved in Hippo signaling pathway, Platelet activation, Osteoclast differentiation, Hematopoietic cell lineage and Leukocyte transendothelial migration (Figure 9 C).

\section{Discussion}

Hypertrophic cardiomyopathy, a genetically heterogeneous disorder of cardiac myocytes, has 


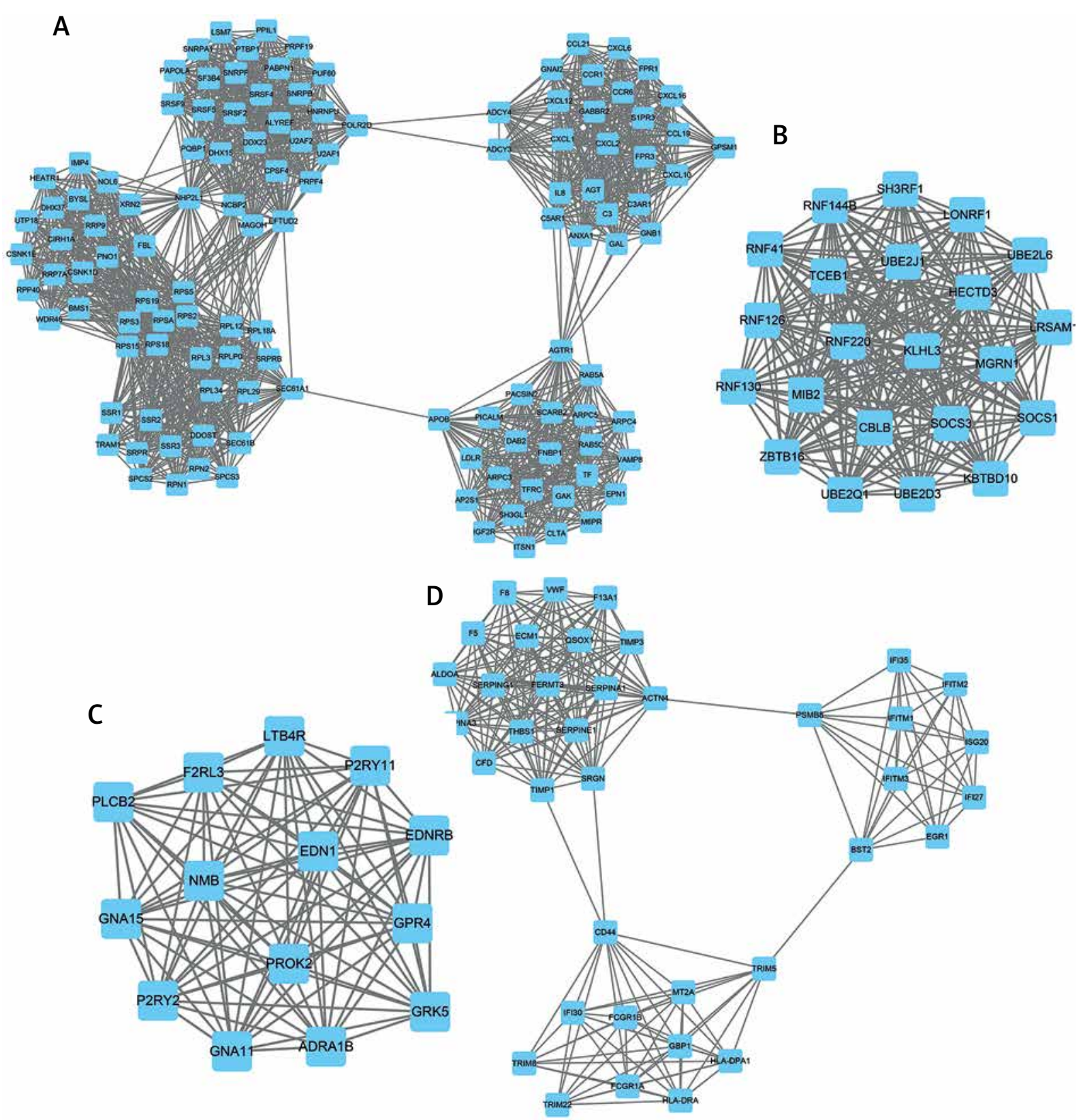

Figure 5. Hub-networks analysis of down-regulated DEGs in HCM. A - Hub-network 1 held 122 nodes and 1664 edges. B - Hub-network 2 contained 22 nodes and 231 edges. C - Hub-network 3 had 14 nodes and 90 edges. D - Hub-network 4 had 38 nodes and 245 edges

been one of the main reasons for sudden cardiac death of young people $[20,21]$. However, the mechanism underlying HCM progression remained unclear. In the present study, we screened differentially expressed proteins and IncRNAs in HCM by analyzing a public dataset (GSE36961). A total of 3127 mRNAs were up-regulated and 3021 mRNAs were down-regulated in HCM. Meanwhile, we also identified 67 up-regulated IncRNAs and 59 down-regulated IncRNAs in HCM compared to control samples. Additionally, protein-protein interaction network analysis was performed to reveal the potential function of DEGs in HCM.
Furthermore, we performed co-expression analysis to reveal the potential roles of differentially expressed IncRNAs.

Previous studies revealed that several genes and pathways were associated with HCM progression. For example, sarcomeric genes (such as MYH7, MYBPC3, TPM1, TNNT2 and TNNI3) are mutated in approximately $40 \%$ of HCM patients [22]. Meanwhile, gene mutation of several pathways, including the RAS signaling pathway, and hypoxia-inducible factor pathway, were also observed in HCM [23]. However, there was still an urgent need to identify novel regulators to elucidate the 
A Control HCM

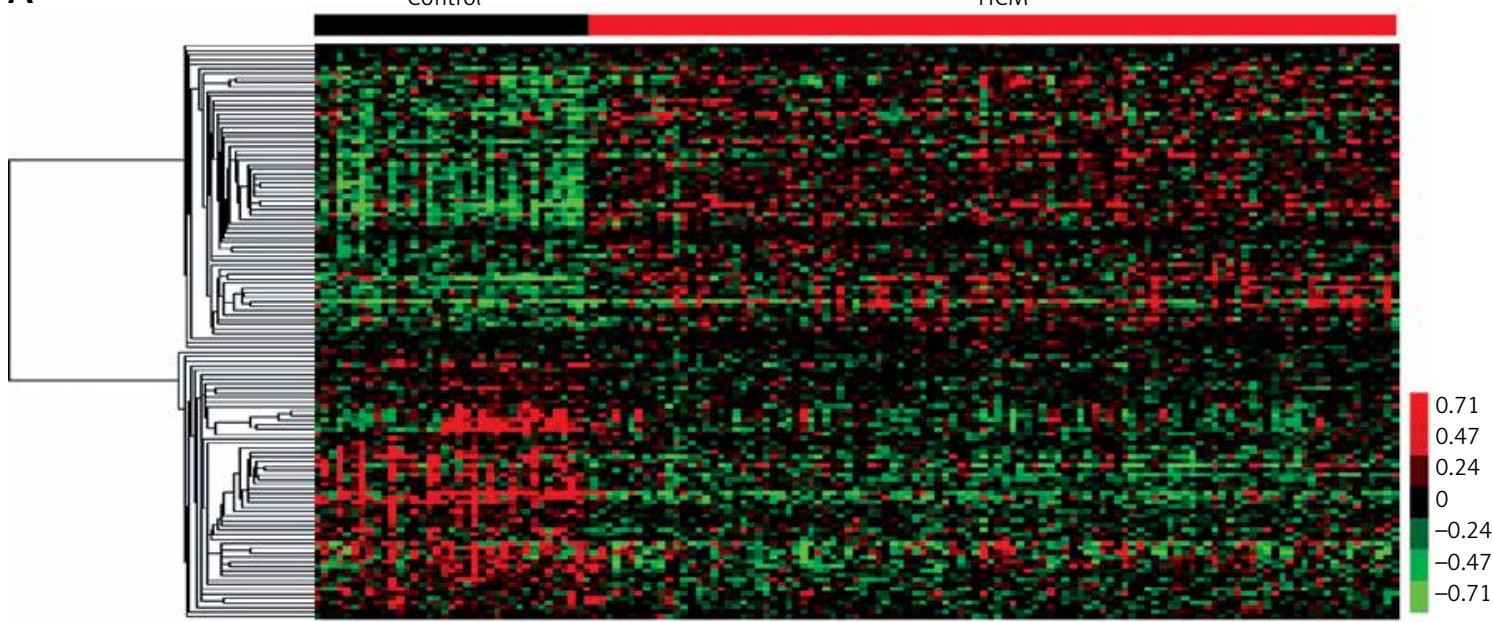

B Biological processes of differently expressed IncRNA in HCM

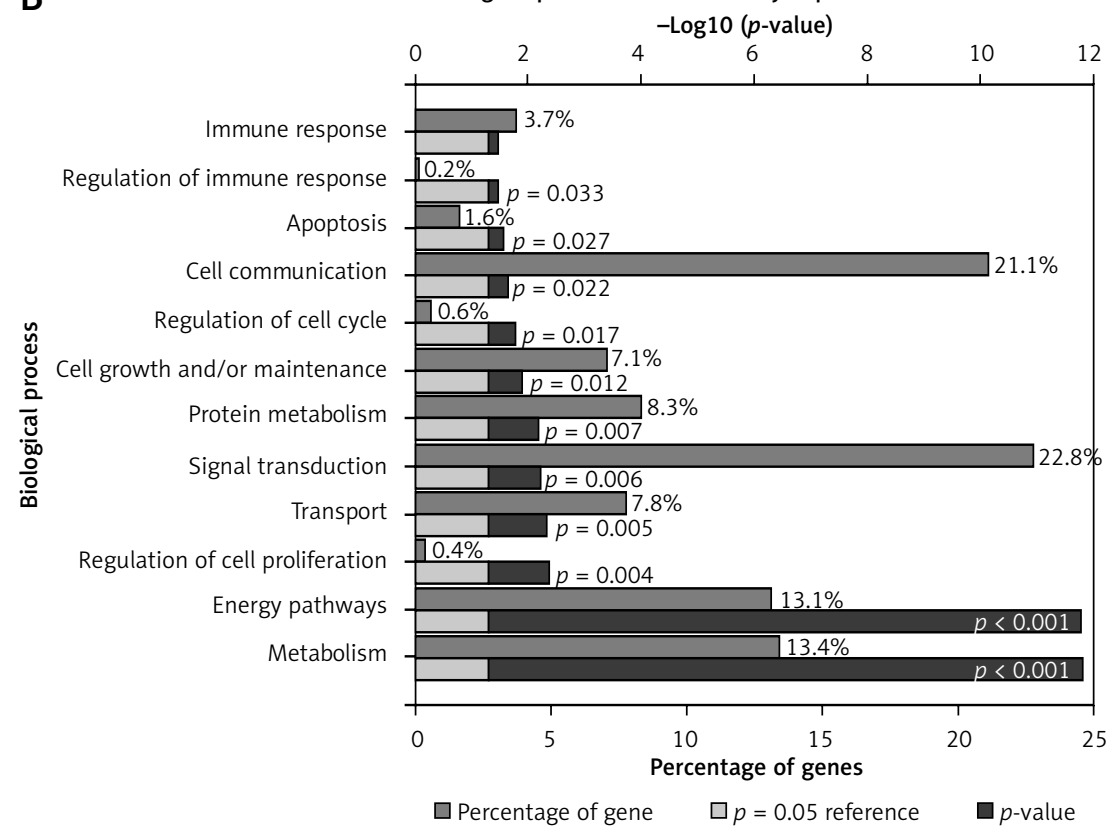

Figure 6. Identification of differentially expressed IncRNA in HCM. A - Hierarchical clustering analysis shows differential IncRNA expression in HCM by using GSE36961. Differential IncRNAs expression analysis shows 67 up-regulated IncRNAs and 59 down-regulated IncRNAs in HCM. B - GO analysis shows dysregulated IncRNA associated biological processes in HCM

molecular mechanisms for HCM. Here, we identified 6158 DEGs in HCM. GO analysis showed that the up-regulated DEGs were mainly involved in regulating Regulation of nucleobase metabolism, Metabolism, Energy pathways, Muscle contraction, Cell growth and/or maintenance, and Protein metabolism. Meanwhile, the down-regulated DEGs were associated with Signal transduction, Cell communication, Immune response, Metabolism, Energy pathways, and Transport. Here, we also constructed HCM associated PPI networks. Several proteins (including down-regulated IL-6, JUN, ACTB, MAPK3, and MYC and up-regulated
UBB, POLR2C, POLR2L, POLR2B, and POLR2G) were found to play more important roles in HCM and interacted with more than 50 proteins. Of note, by screening publications, several genes, such as MYC, have been reported to be involved in HCM progression. For example, emerging studies have shown that inhibition of MYC was becoming an attractive paradigm for prevention and treatment of cardiomyopathy and heart failure [24, 25].

LncRNA, a class of non-coding RNA molecules with more than 200 bases, playsimportant regulatory roles in the transcription, post-transcription and translation regulation of protein-coding genes [7]. 


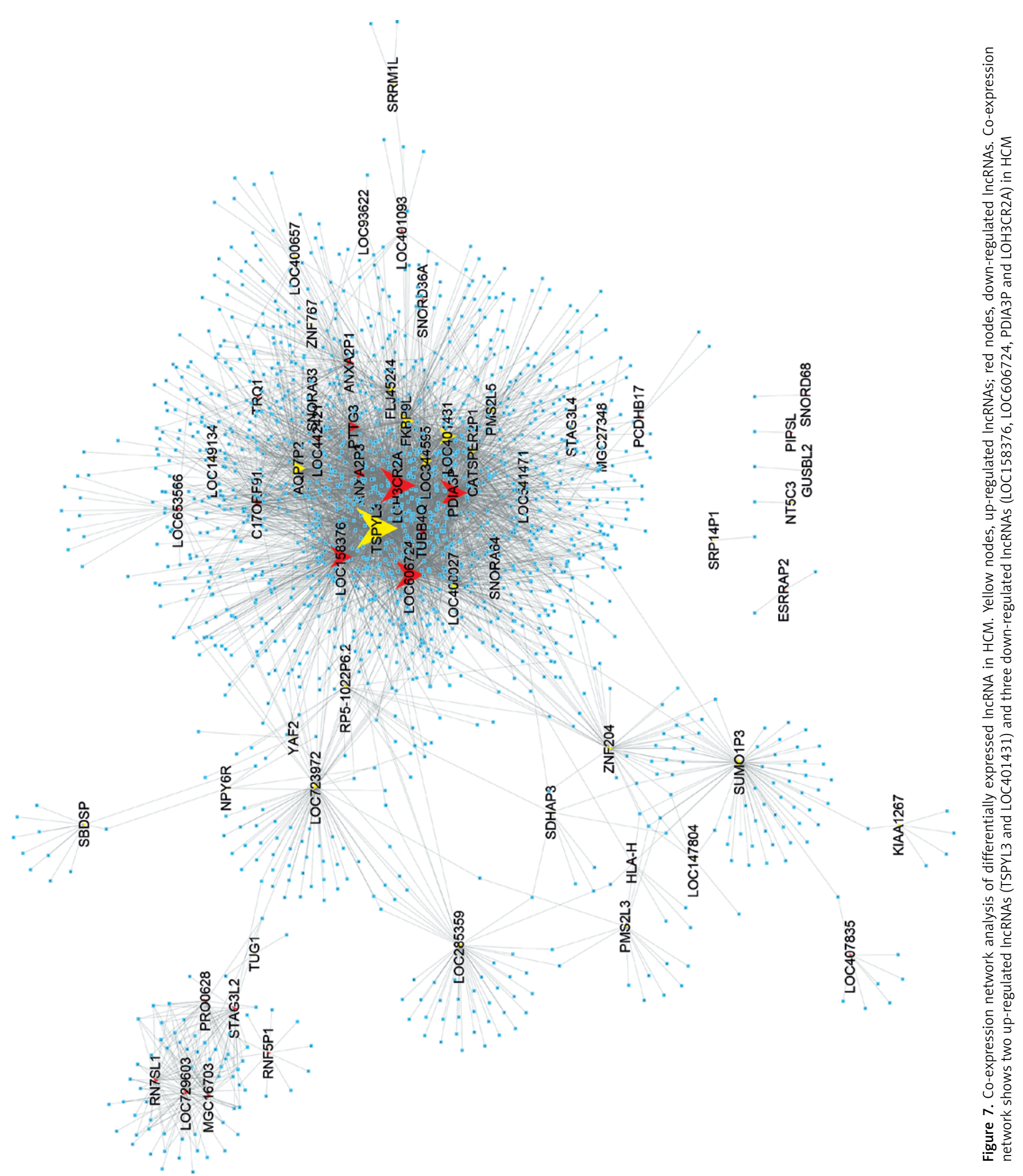


A

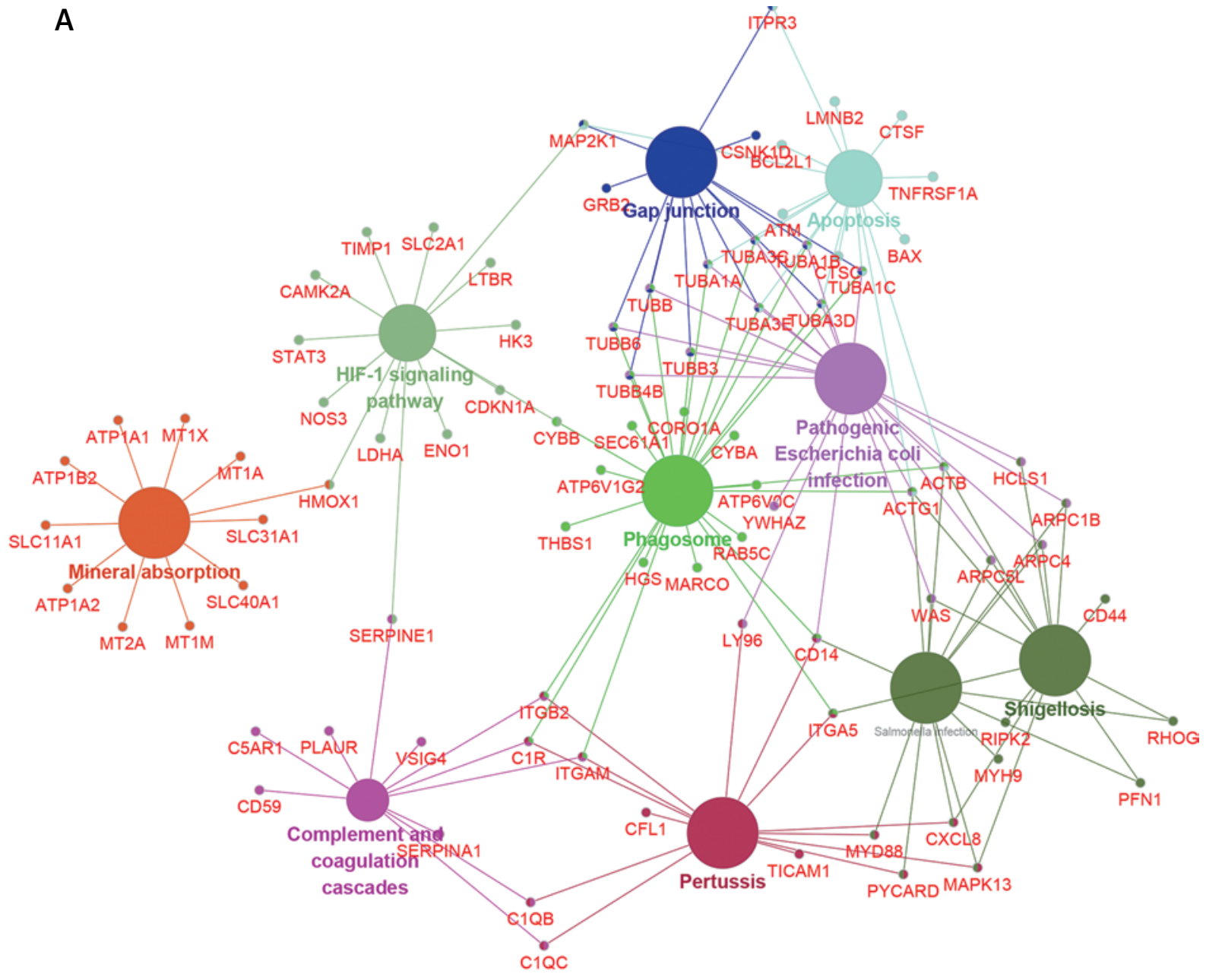

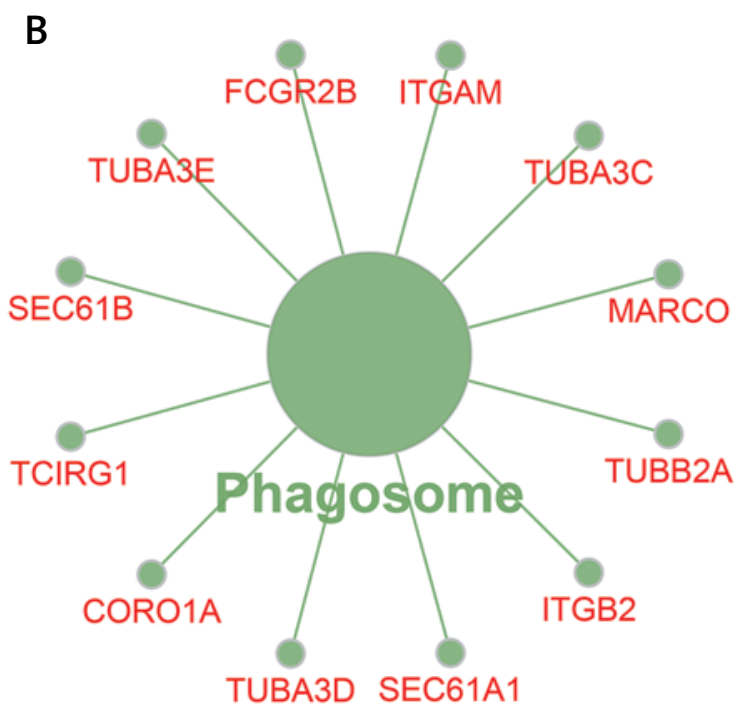

Previous studies have revealed that IncRNAs also regulate cardiomyopathy progression. For example, down-regulation of IncRNA KCNQ1OT1 protects against myocardial ischemia/reperfusion injury following acute myocardial infarction [26].
Figure 8. Molecular function analysis of key up-regulated IncRNAs in HCM. The KEGG pathway analysis shows the up-regulated IncRNA associated pathways. The squares indicate IncRNAs, the arrow-headed nodes indicate mRNAs and the circles indicate pathways

Zhang et al. also reported that the long non-coding RNA H19 promotes cardiomyocyte apoptosis in dilated cardiomyopathy [27]. In the present study, we found that 67 IncRNAs were up-regulated and 59 IncRNAs were down-regulated in HCM. 
A
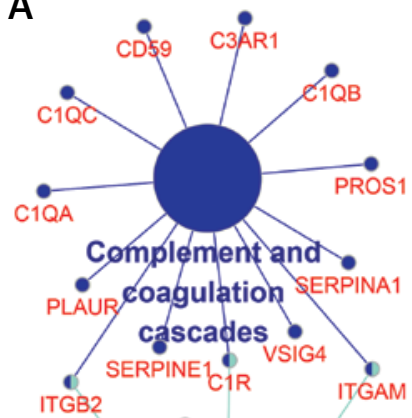

COROPAT TUBA3E

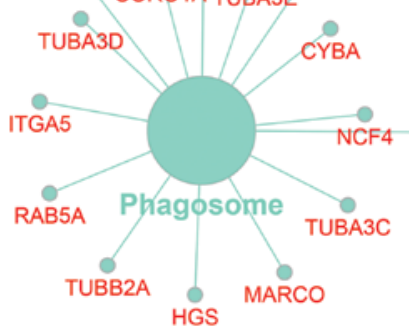

C
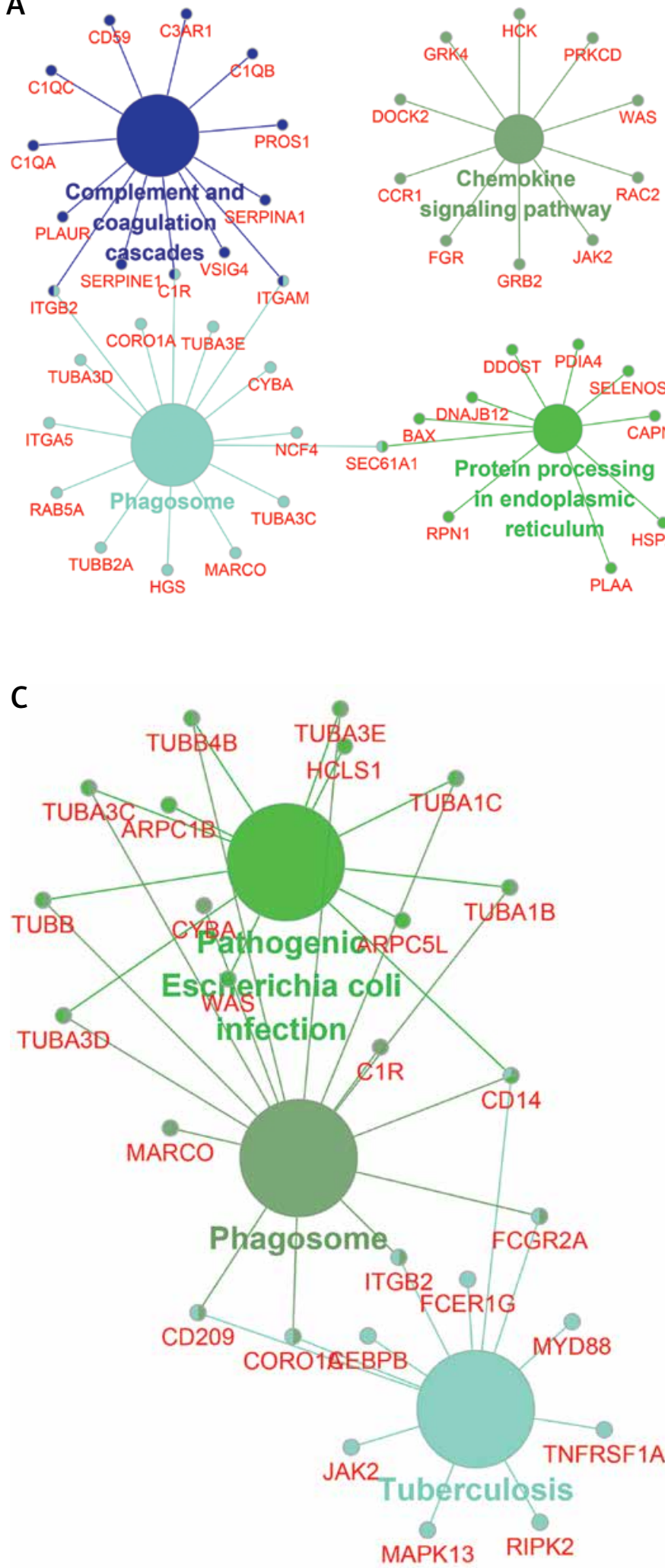

B
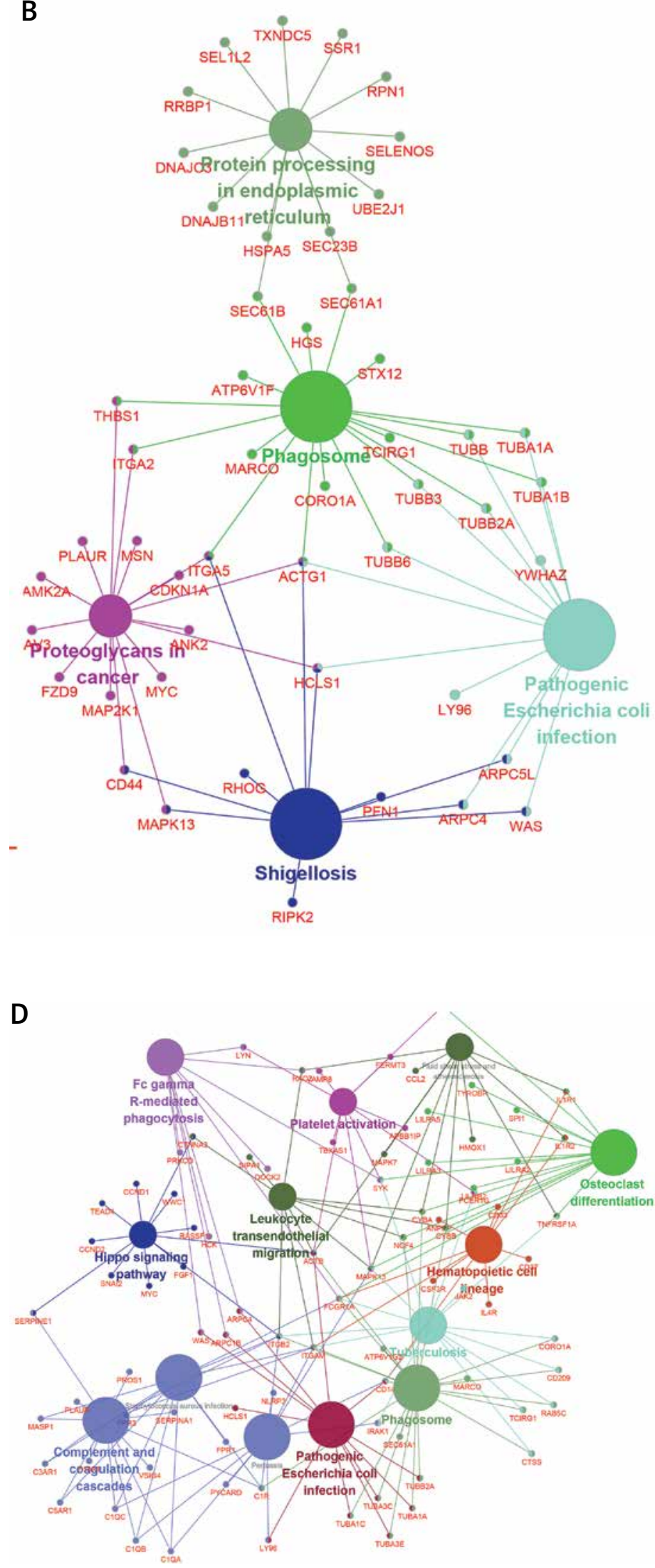

Figure 9. Molecular function analysis of key down-regulated IncRNAs in HCM. The KEGG pathway analysis shows the down-regulated IncRNA associated pathways. The squares indicate IncRNAs, the arrow-headed nodes indicate mRNAs and the circles indicate pathways 
Bioinformatics analysis showed that the dysregulated IncRNAs were mainly involved in regulating Metabolism, Energy pathways, and Regulation of cell proliferation.

Although Kitow et al. and Yang et al. found that IncRNAs could serve as biomarkers for HCM patients, the molecular function of IncRNAs in HCM remained largely unclear. In a co-expression network, we identified TSPYL3, LOC401431, LOC158376, LOC606724, PDIA3P and LOH3CR2A as key IncRNAs in HCM, which co-expressed with more than 200 mRNAs. Of note, TSPYL3 showed the most important regulatory roles by co-expressing with 576 proteins. Furthermore, we constructed IncRNA mediated mRNA-KEGG pathway networks by applying ClueGO in Cytoscape software. We observed that TSPYL3 was associated with HIF-1 signaling pathway, Gap junction, Apoptosis, Phagosome, and Mineral absorption. LOC401431 and LOC158376 were found to be involved in Phagosome regulation. PDIA3P was associated with Complement and coagulation cascades, Chemokine pathway, Phagosome and Protein processing in endoplasmic reticulum. LOH3CR2A was associated with Protein processing in endoplasmic reticulum, Phagosome, Shigellosis, and Proteoglycans in cancer. LOC606724 was found to be involved in Hippo signaling pathway, Platelet activation, Osteoclast differentiation, Hematopoietic cell lineage and Leukocyte transendothelial migration. LOC158376 was found to be involved in Phagosome and Tuberculosis. To the best of our knowledge, this is the first study to reveal the potential roles of these IncRNAs in HCM.

The main objective of this study was to provide information on gene expression which could be relevant for diagnosing HCM in some patients. However, several limitations of this study should be noted. First, we could not provide more detailed information to predict whether the results reported in this paper are homogenously or heterogeneously prevalent in the wide range of HCM cases, or whether our results were sensitive and specific in differentiating HCM from normal, because we did not get enough datasets to support these assumptions. Second, further studies are needed to confirm the expression levels of these key genes and IncRNAs in HCM patients. Last, the molecular functions of these key genes and IncRNAs should be further validated in HCM by using loss/gain of function assays.

In conclusion, we analyzed the public dataset GSE36961 to identify differentially expressed IncRNAs and mRNAs in HCM. A total of 6147 mRNAs and 126 IncRNAs were found to be dysregulated in HCM. PPI network and GO analysis showed that these DEGs were associated with metabolism, energy pathways, signal transduction, and cell communication. LncRNA co-expression analysis showed that IncRNAs were also involved in regulating metabolism, energy pathways, and regulation of cell proliferation. Moreover, TSPYL3, LOC401431, LOC158376, LOC606724, PDIA3P and LOH3CR2A were identified as key InCRNAs in HCM. We believe this study will provide information to explore novel molecular target therapy for HCM.

\section{Acknowledgments}

This work is supported by Huzhou Science and Technology Bureau Project (Grant No. 2014GY36), Guilin Shen.

\section{Conflict of interest}

The authors declare no conflict of interest.

\section{References}

1. Chung MW, Tsoutsman T, Semsarian C. Hypertrophic cardiomyopathy: from gene defect to clinical disease. Cell Research 2003; 13: 9-20.

2. Maron BJ, Gardin JM, Flack JM, Gidding SS, Kurosaki TT, Bild DE. Prevalence of hypertrophic cardiomyopathy in a general-population of young-adults - echocardiographic analysis of 4111 subjects in the cardia study. Circulation 1995; 92: 785-9.

3. Davies MJ, Mckenna WJ. Hypertrophic cardiomyopathy pathology and pathogenesis. Histopathology 1995; 26: 493-500.

4. Maron MS, Olivotto I, Betocchi S, et al. Effect of left ventricular outflow tract obstruction on clinical outcome in hypertrophic cardiomyopathy. N Engl J Med 2003; 348: 295-303.

5. Girolami F, Ho CY, Semsarian C, et al. Clinical features and outcome of hypertrophic cardiomyopathy associated with triple sarcomere protein gene mutations. J Am Coll Cardiol 2010; 55: 1444-53.

6. Baertling F, van den Brand MAM, Hertecant JL, et al. Mutations in COA6 cause cytochrome c oxidase deficiency and neonatal hypertrophic cardiomyopathy. Hum Mutat 2015; 36: 34-8.

7. Wang P, Mao BB, Luo W, et al. The alteration of Hippo/ YAP signaling in the development of hypertrophic cardiomyopathy. Basic Res Cardiol 2014; 109: 435.

8. Kornienko AE, Guenzl PM, Barlow DP, Pauler FM. Gene regulation by the act of long non-coding RNA transcription. BMC Biology 2013; 11: 59.

9. Shi XF, Sun M, Liu HB, Yao YW, Song Y. Long non-coding RNAs: a new frontier in the study of human diseases. Cancer Letters 2013; 339: 159-66.

10. Kitow J, Derda AA, Beermann J, et al. Mitochondrial long noncoding RNAs as blood based biomarkers for cardiac remodeling in patients with hypertrophic cardiomyopathy. Am J Physiol Heart Circ Physiol 2016; 311: H707-12.

11. Yang W, Li Y, He FW, Wu HX. Microarray profiling of long non-coding RNA (InCRNA) associated with hypertrophic cardiomyopathy. BMC Cardiovasc Disord 2015; 15: 62.

12. Suresh R, Li X, Chiriac A, et al. Transcriptome from circulating cells suggests dysregulated pathways associated with long-term recurrent events following firsttime myocardial infarction. J Mol Cell Cardiol 2014; 74: $13-21$. 
13. Zhang $X$, Sun S, Pu JK, et al. Long non-coding RNA expression profiles predict clinical phenotypes in glioma. Neurobiol Dis 2012; 48: 1-8.

14. Smyth GK, Limma: Linear Models for Microarray Data, in Bioinformatics and Computational Biology Solutions Using R and Bioconductor. Springer 2005; 397-420.

15. Page RD. TreeView. Glasgow University, Glasgow, UK 2001.

16. Szklarczyk D, Franceschini A, Kuhn M, et al. The STRING database in 2011: functional interaction networks of proteins, globally integrated and scored. Nucleic Acids Res 2011; 39: D561-8.

17. Shannon P, Markiel A, Ozier O, et al. Cytoscape: a software environment for integrated models of biomolecular interaction networks. Genome Res 2003; 13: 2498-504.

18. Bader GD, Hogue CW. An automated method for finding molecular complexes in large protein interaction networks. Bmc Bioinformatics 2003; 4: 2.

19. Assenov Y, Ramirez F, Schelhorn SE, Lengauer T, Albrecht M. Computing topological parameters of biological networks. Bioinformatics 2008; 24: 282-4.

20. Harris MA, Clark J, Ireland A, et al. The Gene Ontology (GO) database and informatics resource. Nucleic Acids Res 2004; 32: D258-61.

21. Corrado D, Basso C, Thiene G. Sudden cardiac death in young people with apparently normal heart. Cardiovasc Res 2001; 50: 399-408.

22. Erdmann J, Daehmlow S, Wischke S, et al. Mutation spectrum in a large cohort of unrelated consecutive patients with hypertrophic cardiomyopathy. Clin Genet 2003; 64: 339-49.

23. Ashrafian H, McKenna WJ, Watkins H. Disease pathways and novel therapeutic targets in hypertrophic cardiomyopathy. Circ Res 2011; 109: 86-96.

24. Wolfram JA, Lesnefsky EJ, Hoit BD, Smith MA, Lee HG. Therapeutic potential of $\mathrm{c}-\mathrm{Myc}$ inhibition in the treatment of hypertrophic cardiomyopathy. Ther Adv Chronic Dis 2011; 2: 133-44.

25. Lee HG, Chen Q, Wolfram JA, et al. Cell cycle re-entry and mitochondrial defects in myc-mediated hypertrophic cardiomyopathy and heart failure. PLoS One 2009; 4: e7172.

26. Gala-Błądzińska A, Żyłka A, Dumnicka P, Kuśnierz-Cabala B, Kaziuk MB, Kuźniewski M. Sterile leukocyturia affects urine neutrophil gelatinase-associated lipocalin concentration in type 2 diabetic patients. Arch Med Sci AMS 2017; 13: 321-7.

27. Zhang YL, Zhang MY, XU WT, Chen JC, Zhou X. The long non-coding RNA H19 promotes cardiomyocyte apoptosis in dilated cardiomyopathy. Oncotarget 2017; 8: 28588-94. 